\title{
The cool and warm molecular gas in M82 with Herschel-SPIRE
}

\section{J. Kamenetzky ${ }^{1}$, J. Glenn ${ }^{1}$, N. Rangwala ${ }^{1}$, P. Maloney ${ }^{1}$,} M. Bradford ${ }^{2}$, C. D. Wilson ${ }^{3}$, G. J. Bendo ${ }^{4}$, M. Baes ${ }^{5}$ A. Boselli ${ }^{6}$, A. Cooray $^{7}$, K. G. Isaak ${ }^{8}$, V. Lebouteiller ${ }^{9}$, S. Madden ${ }^{9}$, P. Panuzzo ${ }^{9}$, M. R. P. Schirm ${ }^{3}$, L. Spinoglio ${ }^{10}$ and R. $\mathbf{W u}^{9}$

${ }^{1}$ U. Colorado, Boulder, ${ }^{2}$ JPL/NASA, ${ }^{3}$ McMaster U., ${ }^{4}$ UK ALMA, ${ }^{5}$ Universiteit Gent, ${ }^{6}$ LAM Marseille, ${ }^{7} \mathrm{UC}$, Irvine, ${ }^{8} \mathrm{ESA}$ Astrophysics Mission Div.,${ }^{9}$ Laboratoire AIM, ${ }^{10} \mathrm{INAF}$

\begin{abstract}
We present Herschel-SPIRE imaging spectroscopy (194-671 $\mu \mathrm{m})$ of the bright starburst galaxy M82. We use RADEX and a Bayesian Likelihood Analysis to simultaneously model the temperature, density, column density, and filling factor of both the cool and warm components of molecular gas traced by the entire CO ladder up to $\mathrm{J}=13-12$. The high-J lines observed by SPIRE trace much warmer gas $(\sim 500 \mathrm{~K})$ than those observable from the ground. The addition of ${ }^{13} \mathrm{CO}$ (and [C I]) is new and indicates that [C I] may be tracing different gas than ${ }^{12} \mathrm{CO}$. At such a high temperature, cooling is dominated by molecular hydrogen; we conclude with a discussion on the possible excitation processes in this warm component. Photon-dominated region (PDR) models require significantly higher densities than those indicated by our Bayesian likelihood analysis in order to explain the high-J CO line ratios, though cosmic-ray enhanced PDR models can do a better job reproducing the emission at lower densities. Shocks and turbulent heating are likely required to explain the bright high-J emission.
\end{abstract}

Keywords. galaxies: individual (M82), galaxies: starburst, ISM: molecules
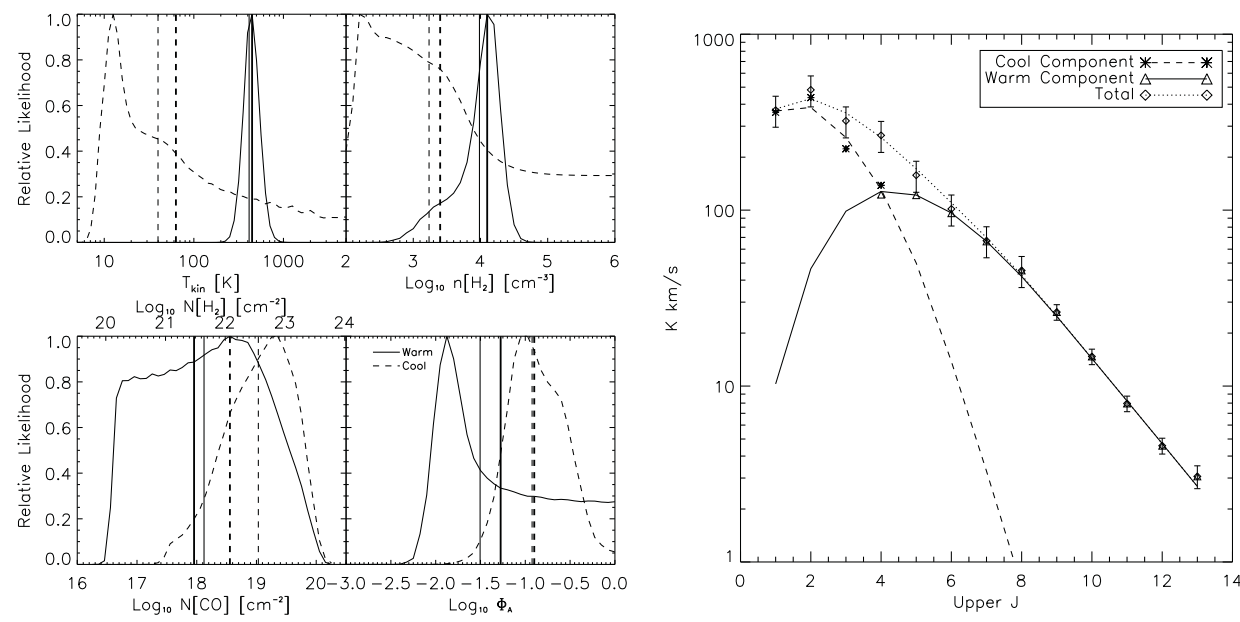

Figure 1. Left: Likelihood analysis of ${ }^{12} \mathrm{CO}$ only, marginalized results. Regular/thick vertical line $=$ median/best fit. Right: Best fit spectral line energy distributions.

\section{Reference}

Kamenetzky, J., Glenn, J., Rangwala, N., et al. 2012, ApJ 753, 70 